Images du travail, travail des images

\title{
L'appareil photo entre les mains d'auxiliaires de puériculture. Recherche-action en images et partage des compétences
}

Giving cameras to child care assistants. Exchange about their professional skils

Irène Jonas

\section{OpenEdition}

Journals

Édition électronique

URL : http://journals.openedition.org/itti/1095

DOI : 10.4000/itti. 1095

\section{Éditeur}

Université de Poitiers

\section{Référence électronique}

Irène Jonas, «L'appareil photo entre les mains d'auxiliaires de puériculture. Recherche-action en images et partage des compétences », Images du travail, travail des images [En ligne], 3| 2017, mis en ligne le 01 février 2017, consulté le 14 avril 2021. URL : http://journals.openedition.org/itti/1095 ; DOI : https://doi.org/10.4000/itti.1095

Ce document a été généré automatiquement le 14 avril 2021

Images du travail, travail des images 


\section{L'appareil photo entre les mains d'auxiliaires de puériculture. Recherche-action en images et partage des compétences}

Giving cameras to child care assistants. Exchange about their professional skils

Irène Jonas

En 2004, le service des crèches du Conseil Général (CG) 93 s'inquiète du trouble identitaire que ressentent les auxiliaires de puériculture et nous sollicite, Liane Mozère $^{1}$ et moi-même, pour approfondir cette question. La démarche du CG 93 est alors d'œuvrer à une revalorisation du métier d'auxiliaire et de travailler à l'émergence des compétences professionnelles des auxiliaires. Face à cette demande, nous faisons le choix sociologique d'initier une recherche-action, à savoir de mettre en place des réunions collectives d'auxiliaires volontaires, sans hiérarchie, démarche qui nous semblait plus porteuse d'un partage des pratiques et d'un échange entre professionnelles qui n'ont pas l'occasion de se rencontrer à moins de travailler dans la même crèche ${ }^{2}$. L'objectif étant de créer un cadre de travail propice à une élaboration commune dans la mise à jour des compétences.

«Ca fait treize ans que je travaille en crèche, je suis venue dans l'optique d'échanger avec d'autres personnes parce que je trouve que nous auxiliaires on n'a pas beaucoup de réunions et on a du mal à échanger sur notre métier, de savoir comment ça se passe dans d'autres crèches, ce qui va, ce qui ne va pas et d'éventuellement trouver des solutions. Aussi parce que je pense que notre métier reste très méconnu. »

\section{Vers une recherche-action-images}

2 La finalité d'une recherche-action consiste en une implication réciproque des acteurs dans la recherche et des auteurs dans l'action, afin d'induire un rapport actif et co- 
construit aux savoirs et à la réalité. Cette approche, qui répond à l'exigence d'établir un lien entre la recherche et l'action, entre la théorie et la pratique, entre la logique du chercheur et celle des acteurs, nous semblait être la mieux à même pour appréhender la communauté "auxiliaires de puériculture " dans son contexte (la crèche), pour tenter de comprendre la signification et les implications du problème et surtout pour réfléchir de manière collective à des solutions.

3 L'objectif était d'accéder à la richesse enfouie au cœur même de la pratique des auxiliaires et qu'elles-mêmes considéraient comme peu de chose ou, pire comme «naturel ». Grâce à la continuité des échanges, elles ont pu construire un dialogue transversal entre elles, par l'intermédiaire de notre seul rôle de " passeuses ». Loin des habituels face-à-face (moi et les autres) se sont progressivement construits des accords provisoires qui faisaient changer tout le monde et ouvraient de nouveaux possibles pour toutes.

«Ainsi, le parti de donner à voir et à entendre la richesse, la complexité et l'inventivité de leur pratique a pu se déployer grâce, non à des qualités qui nous auraient été propres en tant que sociologues, mais bien plus par le biais d'une posture de recherche qui prend d'abord et toujours appui sur ce qui s'énonce du point de vue des personnes interviewées. C'est cette posture, non objectivante mais susceptible d'accueillir l'inattendu, l'impensable, voire l'impossible qui a été le fil rouge dans cet accompagnement. » (Jonas, Mozère, 2006)

4 Dans la recherche classique, tout comme dans la recherche sociologique avec des images, le chercheur est toujours chargé de la rédaction finale du rapport d'enquête et le chercheur/photographe de la production d'images. Si la prise de vues sur le terrain nous apparaît, certes, être un outil de recherche pertinent apte à récolter des données et à analyser des phénomènes sociaux (Maresca, Meyer, 2013, 26), il y allait de la crédibilité de la recherche-action que l'écriture et le travail de production et d'analyse des images soient collectifs (Jonas, Mozère, 2011).

5 Le choix de laisser l'appareil photo entre les mains des acteurs n'est pas nouveau, mais il a le plus souvent été réalisé, notamment au cours des années 1980-1990, non dans le cadre de recherches sociologiques, mais dans celui d'actions culturelles menées dans les quartiers " en difficulté " ${ }^{3}$. L'objectif était alors de donner la parole et l'image à des populations stigmatisées, la photographie constituant pour ces publics un art accessible propre à favoriser l'émergence de regards pluriels, une ouverture sur le monde et sur eux-mêmes, une déconstruction des clichés et une accession à la citoyenneté (Jonas, 1998). L'association « Paroles par l'Image », créée en 2007, et l'association « Regarde ! ", créée en 2012, s'inscrivent dans une approche identique en voulant donner à des publics vulnérables le désir et la capacité de s'exprimer par l'image. D'autres expériences innovantes avec la photographie ont eu lieu en sociologie, comme les autoportraits d'agricultrices étudiés par Sylvain Maresca, mais souvent sans que le projet sociologique n'inclue à la fois la prise de vue et la réception des images par les mêmes acteurs.

6 Le parti pris de demander aux auxiliaires de réaliser elles-mêmes les clichés répond ainsi à un double objectif. Inscrire la photographie dans la démarche collective propre à la recherche-action mais également permettre aux auxiliaires de produire elles-mêmes des représentations visuelles de leur métier, compte tenu de la difficulté à définir leurs compétences professionnelles qu'elles vivaient davantage comme des qualités féminines. 
7 En effet, les auxiliaires ont une mauvaise image d'elles. Elles se sentent peu écoutées et ont souvent de grandes difficultés à parler de leurs compétences dans la mesure où leur métier n'est pas valorisé. Il nous semblait que leur faire la proposition de réaliser des images, que nous appellerons "extérieures" pour les distinguer de leurs images « intérieures », pouvait permettre d'approcher et d'analyser les images «intérieures » qu'elles avaient de leur métier. Il nous était en effet apparu au cours de la première partie de la recherche-action que les auxiliaires exprimaient la sensation de faire les choses « naturellement ", plus parce qu'elles étaient femmes, donc naturellement aptes à s'occuper des enfants, que parce qu'elles avaient acquis de «vraies » compétences professionnelles. Il leur était donc très difficile d'énoncer en quoi leur pratique quotidienne relevait de savoir-faire et de savoir-être professionnels. Pour une autre part, les auxiliaires se présentaient comme ayant « le nez dans le guidon». Le fait de prendre des photos, nous semble-t-il, leur demanderait un certain recul, une sorte de temps « d'arrêt sur image » et les obligerait en quelque sorte à réfléchir sur ce qui leur paraissait important de montrer et donc d'énoncer par la suite.

De plus, au cours de la première année de la recherche-action, nous nous étions aperçues que l'échange se faisait plutôt autour des phrases telles que «les enfants sont plus violents ", " on n'est pas respectées » et que lorsqu'on demandait aux auxiliaires d'illustrer leur propos, elles avaient souvent du mal à donner des exemples précis. Il nous a alors semblé que l'utilisation de la photographie, de leurs photographies, pouvait permettre non seulement de partir d'une situation, voire d'une anecdote, pour arriver au général, mais également aux autres auxiliaires de compléter, d'enrichir ou de questionner par d'autres situations qu'elles-mêmes avaient vécues ou photographiées. À travers leurs propres images et celles des autres, ce sont toutes leurs compétences professionnelles et de réactivité à des situations, tant avec les enfants que les adultes (parents et personnels de crèche) qui leur sont apparues. La photographie, sans faire de jeu de mot, a joué un rôle important de révélateur.

« On fait les choses comme ça, on est tellement dans le faire que ça nous semble naturel. On

fait ça tous les jours."

9 La photographie dans le cadre de la recherche-action, a ainsi permis aux auxiliaires, et c'était là l'enjeu de cette démarche, non seulement de visibiliser les nombreuses compétences dont elles n'avaient pas forcément conscience mais également d'entamer une véritable réflexion sur la professionnalité de leur action qu'elles avaient tendance à présenter d'emblée comme naturelle. Car, comme l'a dit l'une d'entre elles :

"Quand une fille n'est pas douée, on dit qu'elle peut garder des enfants... Mais on "garde" les vaches, pas les enfants. "

\section{Mise en œuvre du projet}

10 Le projet d'intégrer la photographie à la recherche-action n'a pas été initié dès le démarrage de celle-ci. Pendant une année, pour le premier groupe d'auxiliaires, seules des réunions collectives avaient été organisées. C'est au cours des réunions de restitution et en analysant les premiers résultats avec le service des crèches qu'a été envisagé et accepté le projet de photographie participante que nous avions suggéré pour le second groupe. Il nous semblait que l'image pouvait offrir les moyens d'approfondir les différents problèmes soulevés lors de la première session. 
11 La proposition faite aux auxiliaires de réaliser des images a été bien accueillie, mais a immédiatement soulevé des objections d'ordre divers. La première objection évoquée s'est rapportée au manque de temps lié aux contraintes du travail et à l'absentéisme. Outre leurs inquiétudes concernant leur capacité à faire de «belles images" ou à photographier des "choses intéressantes", leurs principales interrogations ont concerné la nécessaire autorisation de la directrice de la crèche ${ }^{4}$, l'information aux parents, l'éventuel refus de leurs collègues à être photographiées, l'inquiétude du lien entre photographie de petits enfants et pédophilie, le droit à l'image. Ces sujets ont donné lieu à des débats dans le collectif et les demandes d'autorisations ont été transmises au service des crèches.

12 Après deux premières réunions, des appareils photographiques jetables ${ }^{5}$ ont donc été confiés aux auxiliaires avec comme consigne de photographier leurs pratiques à la crèche. Délibérément nous avons opté pour une consigne large afin que les auxiliaires puissent se l'approprier. Le rendu des images a d'ailleurs été très diversifié, pour n'en citer que quelques uns : les «mauvaises postures », le déroulé d'une journée, le «travail invisible» (rangement, préparation), des situations particulières, des moments « heureux », l'environnement (vétusté, aménagements inadéquats, etc.), les jeux libres et les activités, les repas, etc.

Les jetables, une fois les photos réalisées, ont été remis au service des crèches du Conseil général qui nous a remis l'intégralité des tirages et des négatifs. Au cours des réunions qui ont suivi, chaque auxiliaire présentait, à ses collègues et nous-mêmes, ses clichés et exposait tant sa démarche générale que les scènes photographiées, le «ce qu'elles avaient voulu montrer à ce moment-là ", images et commentaires étant à leur tour interrogés par les autres auxiliaires.

Cette restitution des images a été d'une grande richesse et s'est souvent déroulée selon un même scénario. Dans un premier temps, la curiosité dominait, en effet, les auxiliaires ne connaissent bien souvent que la crèche où elles travaillent et la découverte des photographies leur permettait de visualiser d'autres structures et d'autres espaces, tant intérieurs qu'extérieurs. Les échanges portaient ainsi en premier lieu sur les différences existantes entre «ma » crèche et « cette » crèche, questions et descriptions s'entremêlant autour des aménagements et des avantages ou contraintes qu'ils génèrent pour l'ensemble des tâches quotidiennes. La photographie a permis ici de faire apparaitre tout un volet de l'organisation informelle que mettent en place les auxiliaires pour le bien-être des enfants et tout particulièrement pour le sommeil qui est un élément important pour la vie en collectivité. 
Photographie 1.

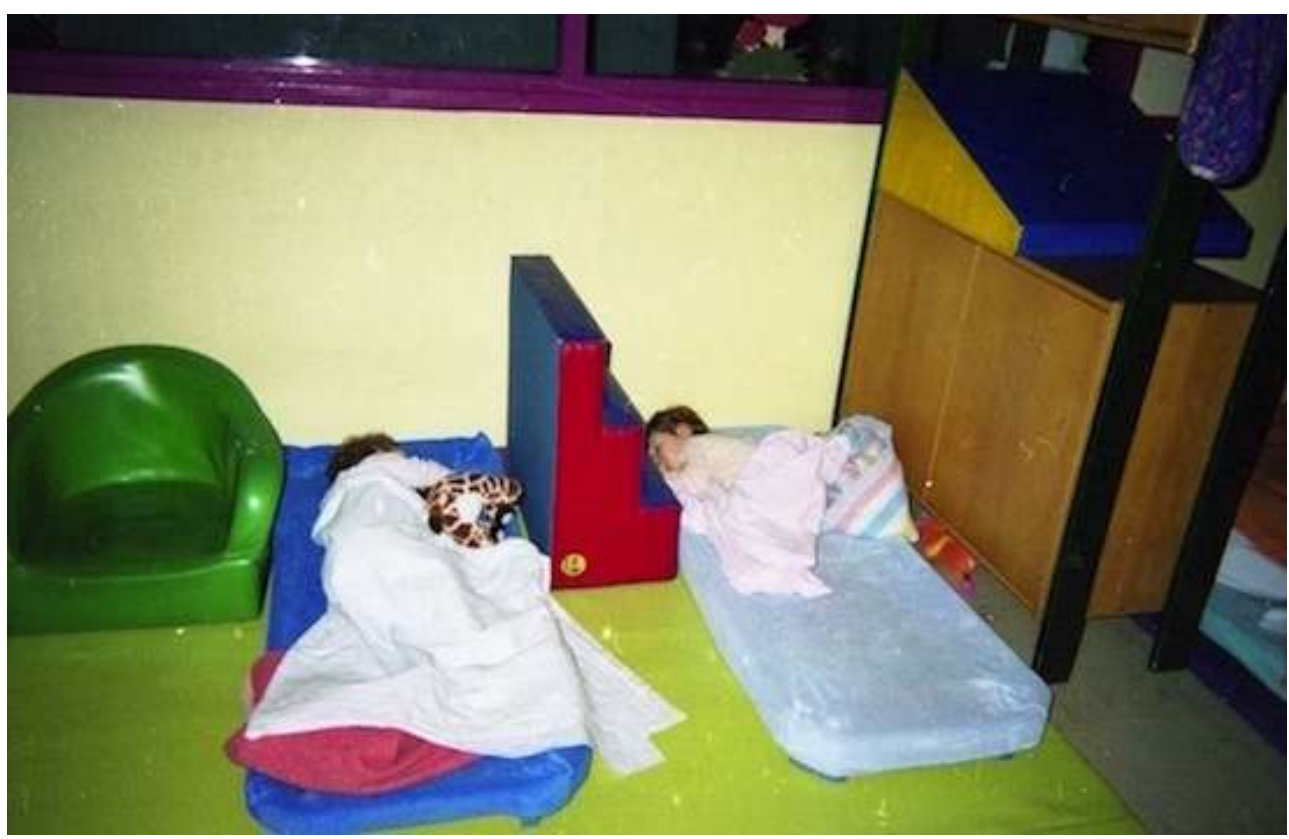

"À la sieste, on sépare les lits par des meubles de façon à ce qu'ils aient chacun un espace.

- Vous n'avez pas de dortoirs?

- Non, parfois on se retrouve à bloquer une salle pour les enfants qui ont besoin de dormir le matin, une salle de jeu.

- Nous non plus, on n'a pas de dortoirs, alors on en a discuté avec l'équipe et on a organisé un coin avec de gros animaux en tissus pour qu'ils puissent se poser au moins trois quart d'heure. "

Dans un second temps l'auxiliaire qui avait disposé comme elle le souhaitait la totalité de ses images sur une table expliquait son projet photographique et la façon dont elle avait procédé.

«Moi j'ai pris des images du quotidien, comment se déroule un repas, un lever de sieste et des situations particulières... justement une petite fille quand elle n'est pas contente, elle boude, ou alors des enfants pendant une dispute pour un jeu, mais il fallait avoir l'appareil toujours sur soi. Quand je quittais la pièce, je l'avais dans la poche. Et dès qu'il y avait quelque chose qui me semblait bien pour moi, crac je faisais une photo."

16 Les partages d'expérience amorcés par les échanges autour des photographies des unes et des autres ont permis une prise de conscience des nombreuses compétences qu'elles développaient dans le cadre de leur travail quotidien, propre à une véritable réflexion sur leur professionnalité. Cette circulation de la parole a été l'élément le plus étonnant et le plus enrichissant dans la mesure ou, d'une séance à l'autre se sont noués, puis dénoués et retissés des liens dans les idées, mais aussi dans les possibles solutions.

Le rapport final a été travaillé avec les auxiliaires, tant pour le choix des exemples que pour celui des photos qu'il leur semblait significatifs d'y intégrer. Une première mouture leur a été remise, puis une réunion a été organisée pour en débattre avant de remettre la version finale au CG 93. 


\section{Les compétences des auxiliaires en images}

18 Si chaque auxiliaire a choisi de photographier ce qui lui apparaissait le plus représentatif de son métier, le recueil des photographies a permis de faire émerger deux grands thèmes: la souffrance au travail (mauvaises postures, aménagements inadaptés, etc.) et les compétences au travail.

Si l'on met de côté les situations particulières, bien que très fréquentes, d'absentéisme et donc de surcharge de travail, ce dont se plaignent avant tout les auxiliaires sont : la manutention permanente, le manque d'adaptation du mobilier, les gestes quotidiens avec les enfants et les douleurs de dos qui en découlent. Pour illustrer ce propos, nous citerons l'exemple d'une auxiliaire qui a choisi de ne photographier à la crèche que ses collègues pour témoigner des mauvaises postures et les réactions de ses collègues.

\section{Les mauvaises postures}

Lorsqu'un espace est conçu pour les tout petits, la taille des meubles leur est totalement adaptée: petits fauteuils, petites chaises, petites barrières, ce qui obligent les auxiliaires à sans cesse être penchées ou mal assises. À cela s'ajoutent encore les multiples jouets à ramasser, les matelas à installer/désinstaller s'il n'existe pas de dortoir, les stores des baies vitrées à baisser/remonter, etc.

Solène, lorsqu'elle s'est retrouvée à la crèche avec l'appareil photo, a choisi de réaliser des images sur les mauvaises postures, parce que dit-elle :

" on a mal au dos, on a tout le temps mal au dos. On va dire que c'est de notre faute, qu'on n'a qu'à bien se baisser, mais quand il y a plein de jouets éparpillés dans une salle... Bien se baisser pour un jeu, d'accord, mais quand il faut ramasser cinquante fois en cinq minutes, on ne plie pas les genoux on se penche automatiquement. » (Photos 2, 3, 4 et 5)

Photographie 2.

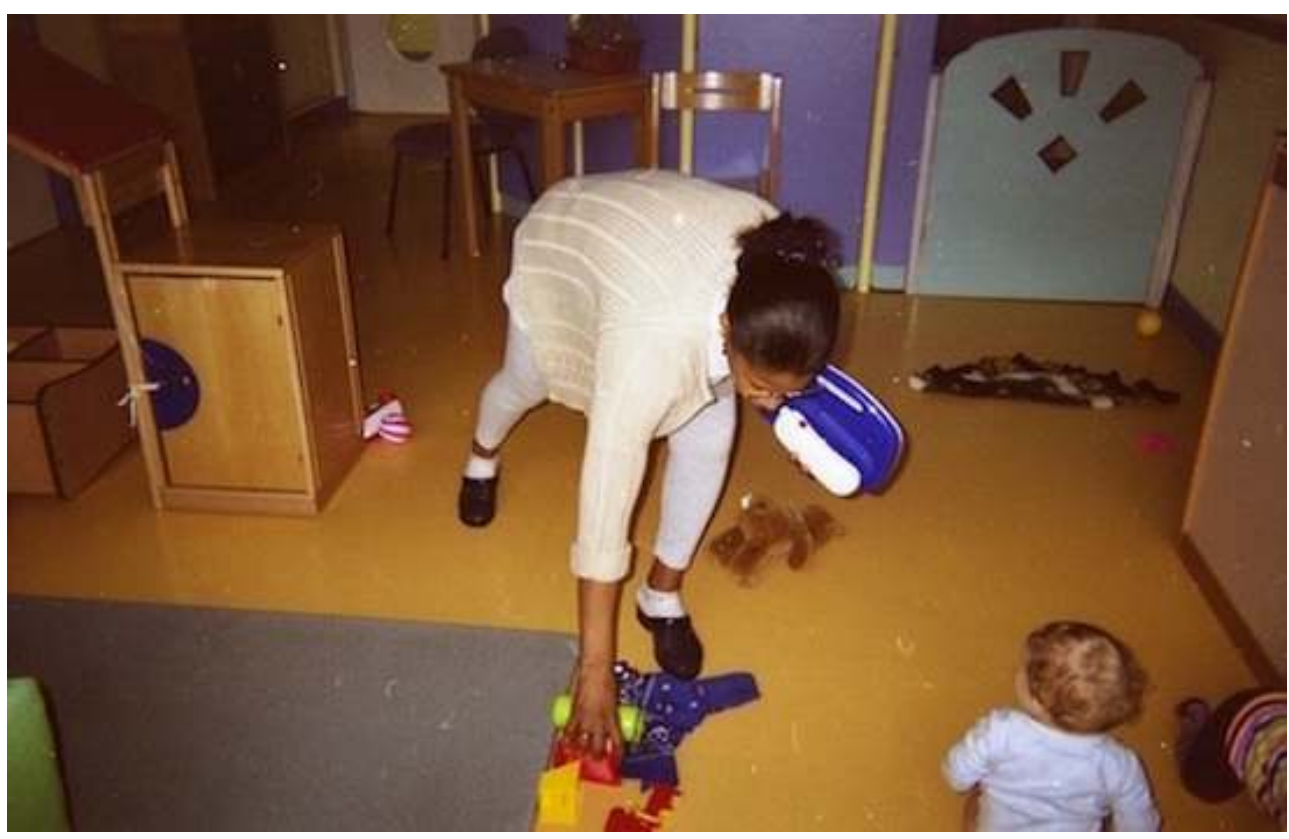


Photographie 3.

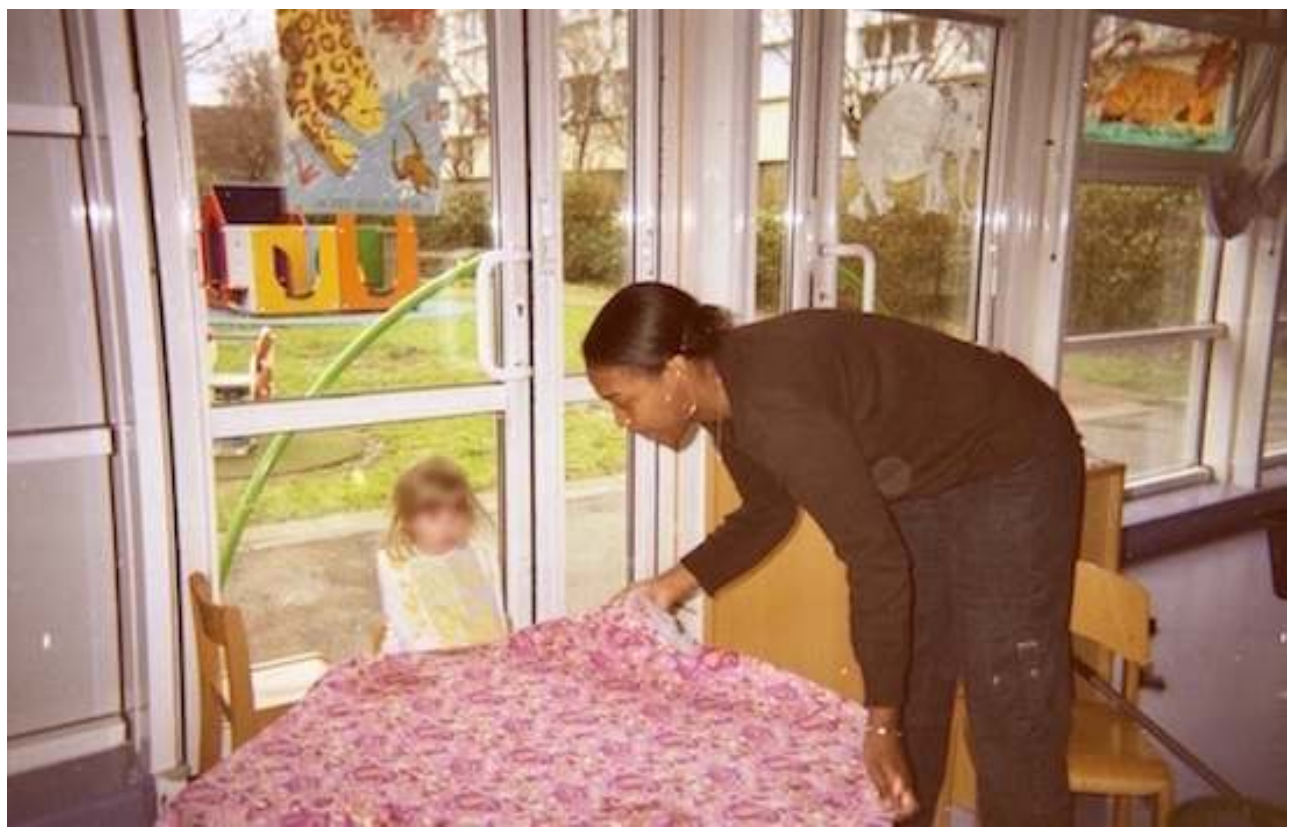

Photographie 4.

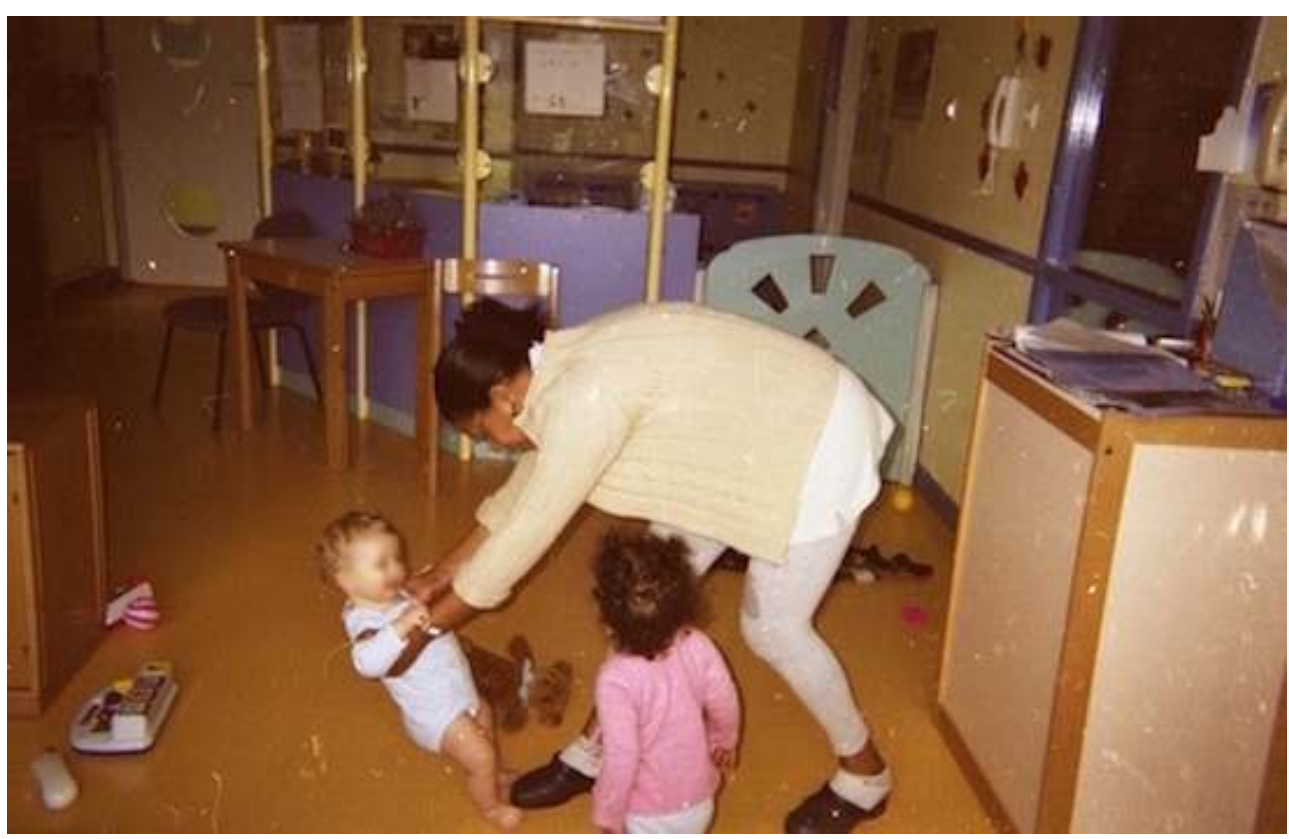


Photographie 5.

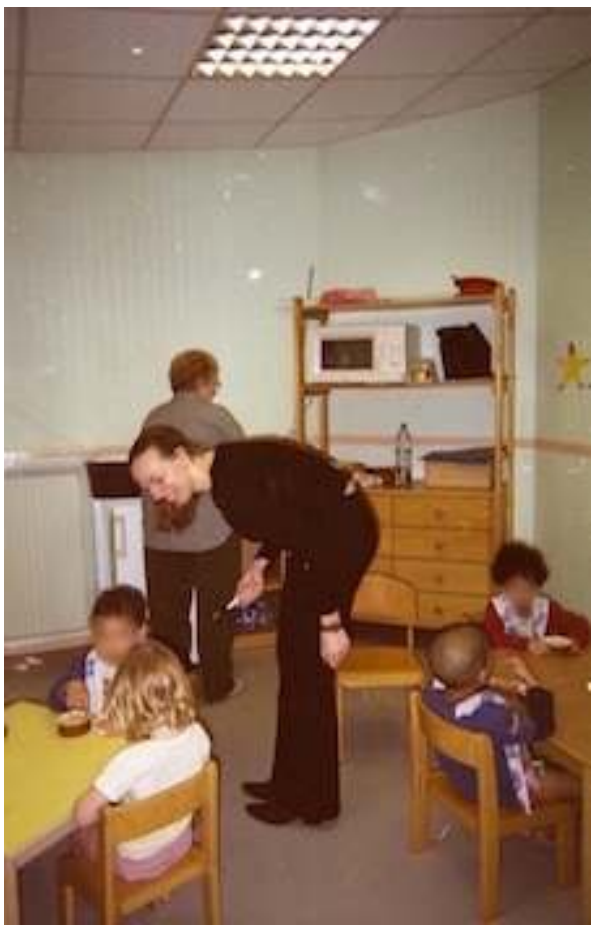

22 Au fur et à mesure que les autres auxiliaires regardent les photographies de Solène, elles appuient ou illustrent son propos par d'autres situations qu'elles connaissent dans leur crèche en montrant leurs images.

"C'est vrai, si on prend le temps de bien se baisser à chaque fois, il nous faut la journée. [...] On ne pense pas forcément à plier les genoux quand on doit se baisser à plusieurs reprises pour ramasser des jeux qui traînent ou pour jouer avec un enfant. [...] Pour le déjeuner, le goûter, on doit chaque fois mettre les tables, débarrasser les tables, elles sont basses. [...] Et toutes les manipulations quand les enfants sont partis pour ranger les salles. » (Photos 6 et 7) 
Photographie 6.

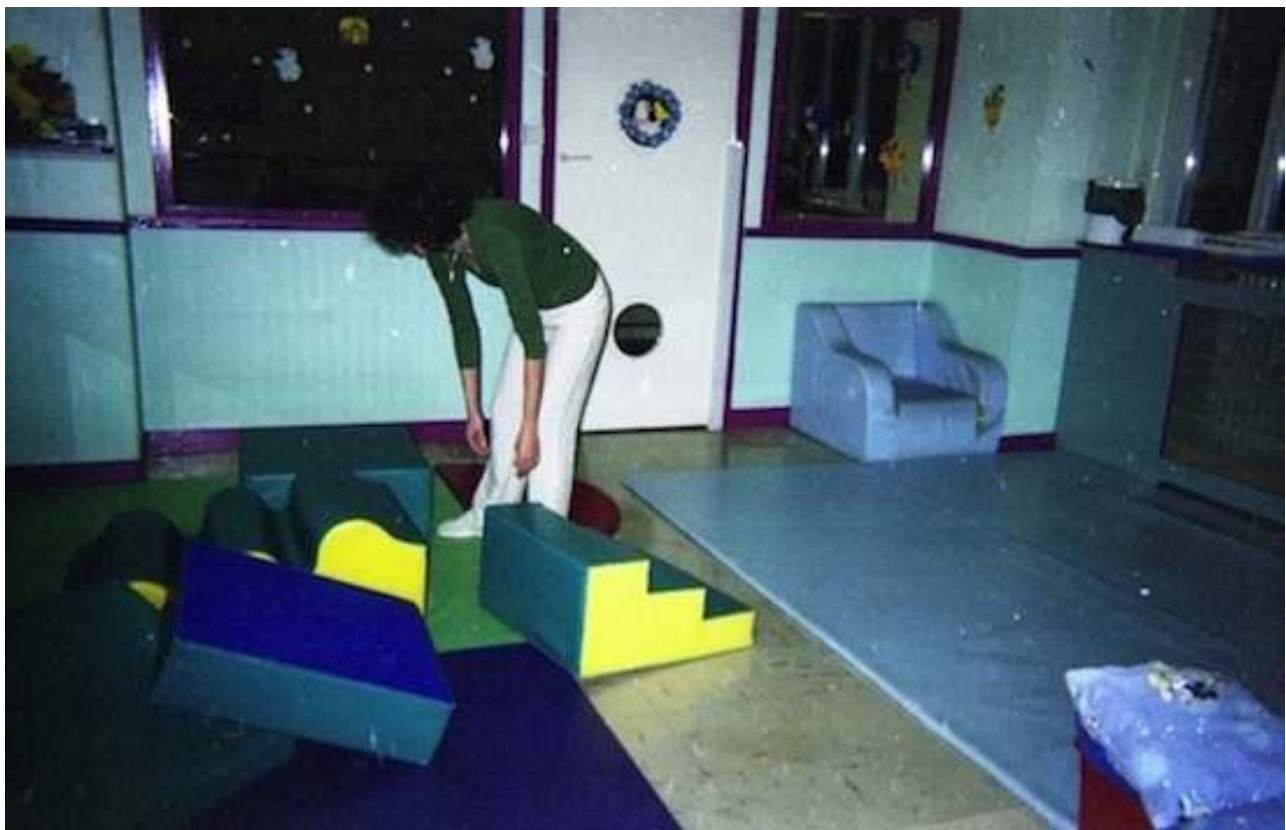

Photographie 7.

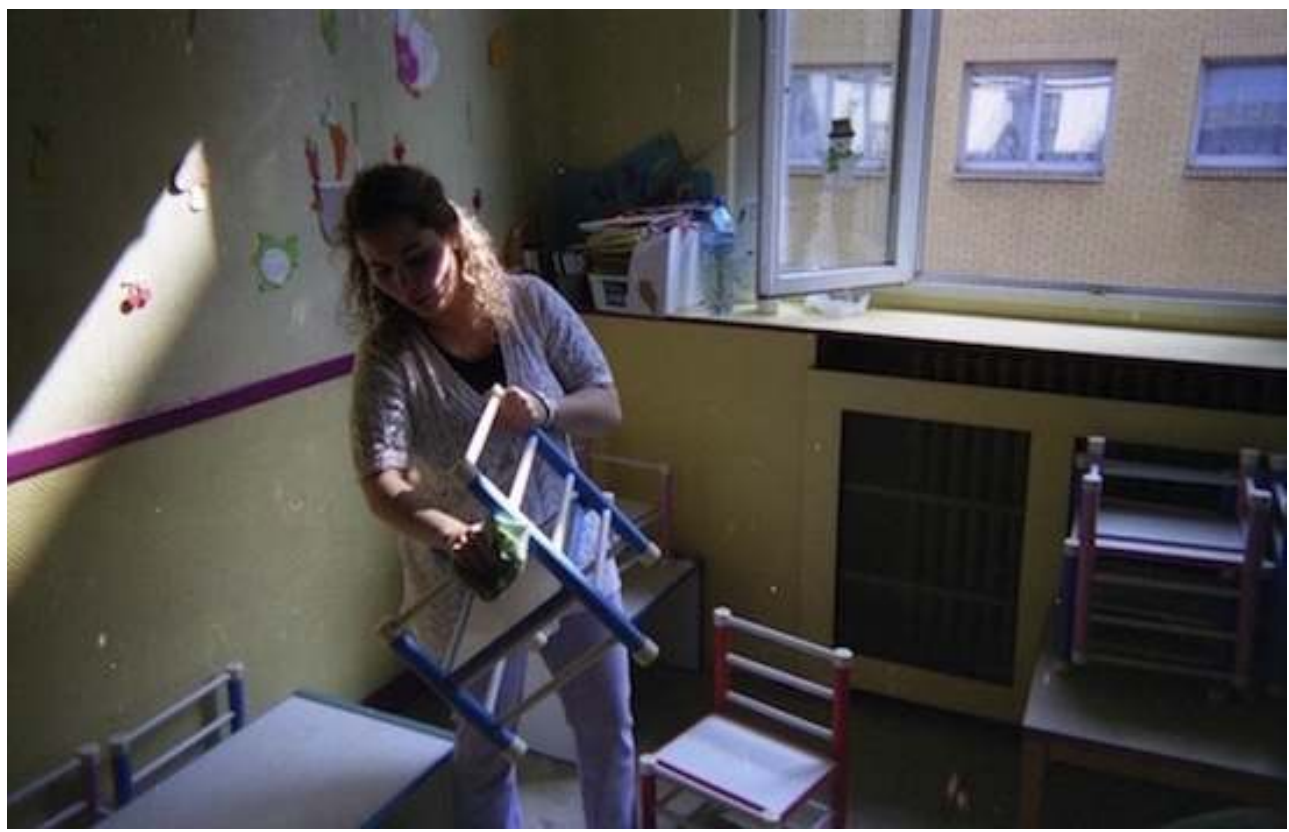

Lorsque c'est le tour de Berthe de présenter ses photos, elle pose le cliché d'un petit escalier près des changes, image qui d'emblée ne signifiait pas grand chose pour nous. Par contre, la réaction ne s'est pas faite attendre de la part des autres auxiliaires :

"J'ai photographié l'escalier parce que quand la crèche a été rénovée, une ergonome est venue et elle nous a beaucoup aidées pour nous éviter de nous fatiguer. L'escalier ça vient de là et c'est bien pratique. En plus ils aiment bien, ça devient un jeu d'aller se changer, ils se cachent en-dessous. Chez nous la salle de bain est toujours fermée, donc il y a toujours un adulte et ça ne risque rien. Et de toutes façons, ils ont très bien compris que si nous on n'y était pas, ils ne doivent pas monter dessus. » 
24 la part des autres auxiliaires :

«C'est super, je ne savais pas qu'on avait le droit. [...] Au niveau des normes de sécurité vous n'avez pas eu de problèmes? [...] «Je ne sais pas si notre directrice accepterait. »

Certaines mentionnent qu'elles aussi ont un escalier mais de forme et de matière différente :

« Nous, on a aussi un escalier mais les enfants ne peuvent pas aller en dessous, le problème c'est qu'il est lourd quand on doit le déplacer » dit Pauline en plaçant une de ses photographies à côté de l'image initiale (photo 8).

Photographie 8.

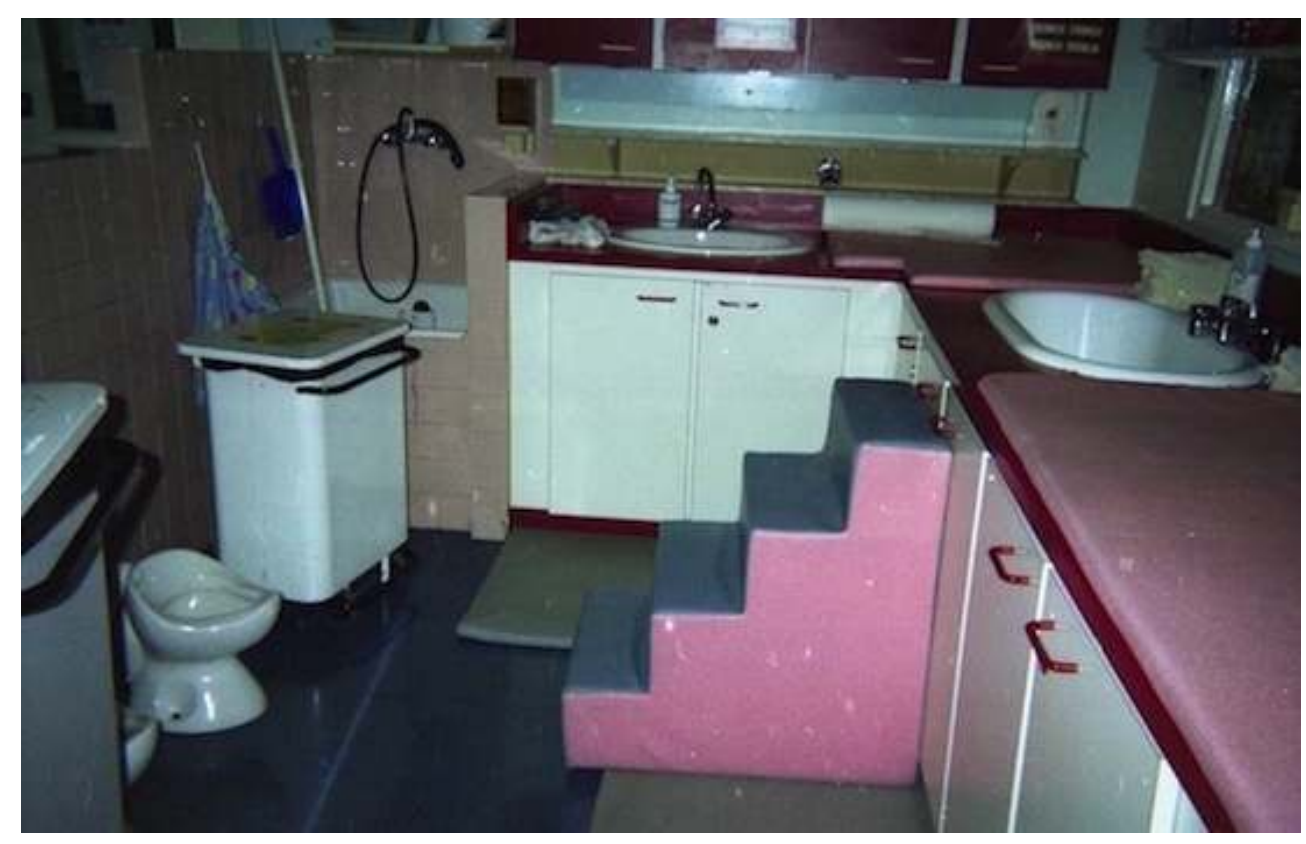

Il apparaît ainsi que chez les moyens et chez certains des grands qui ont encore besoin de couches, le poids des enfants est une donnée importante dans la vie quotidienne de la crèche. Comme l'expliquent alors plusieurs auxiliaires, pour éviter cet effort, elles changent le plus possible les enfants debout. Jocelyne en témoigne immédiatement avec une de ses photos :

«Ca devient pénible et difficile de soulever les enfants mais on est obligé de le faire pour les mettre sur le meuble à langer. Pour ne plus avoir à les porter sur les tables de change qui sont quand même hautes, ou on s'accroupit au sol ou on prend une petite chaise. " (Photo 9). 
Photographie 9.

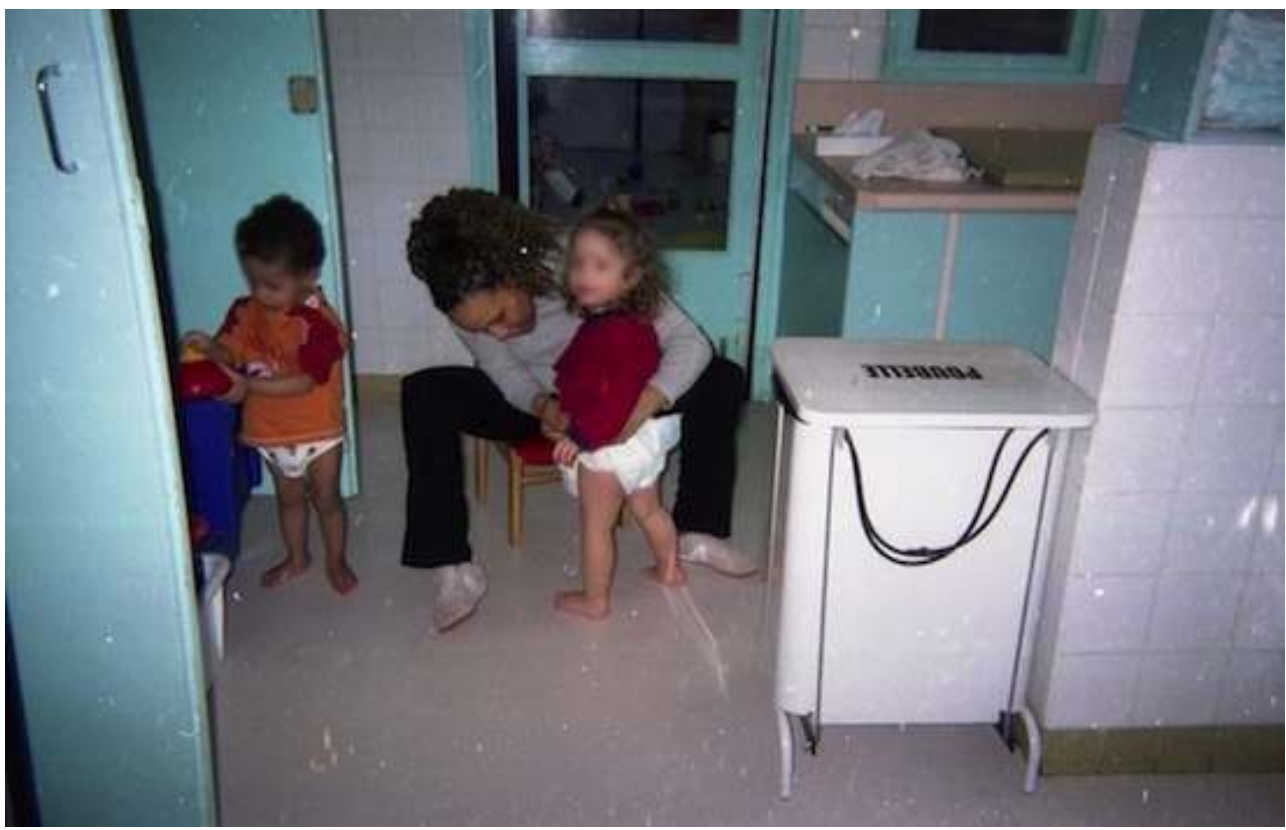

Face à cette image qui semble parler à tout le monde, les réactions fusent et des photographies que nous n'avions pas encore visionnées sont sorties des boîtes:

«Sur la petite chaise on en prend un coup. Le seul moment où on est vraiment bien installée, c'est quand on est à la table d'adulte avec une chaise d'adulte, sinon tout est vraiment petit " dit Aline en sortant de sa boîte une photographie presque identique. (Photo 10).

\section{Photographie 10.}

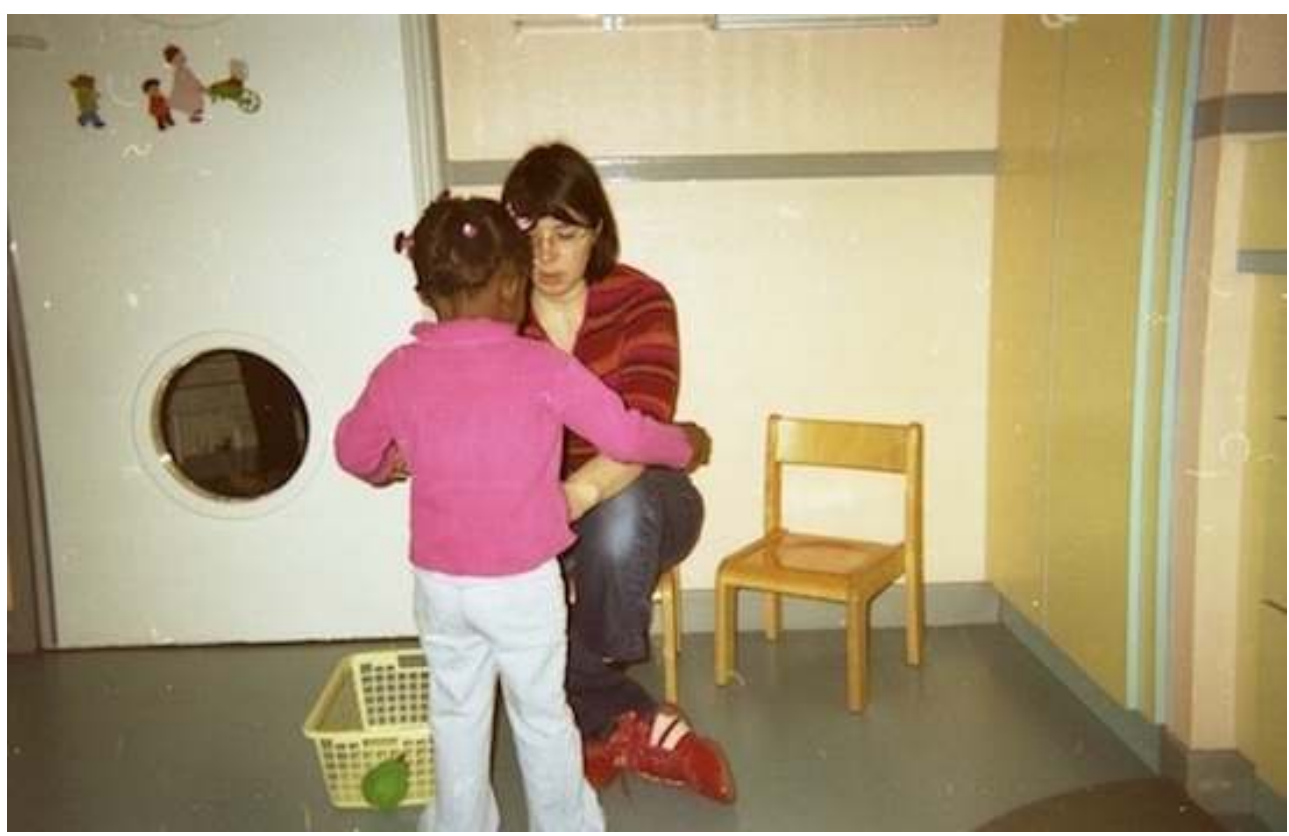

"Même quand on a des meubles à notre taille, ça ne va pas toujours ", reprend une autre en montrant une photo, "Nous on a des grands fauteuils à notre taille d'adulte, ils sont confortables quand on est assis au fond pour donner un biberon, mais pas pour donner à manger à un bébé dans un transat. Ils sont trop mous et on n'est pas à l'aise. » (Photo 11). 
Photographie 11.

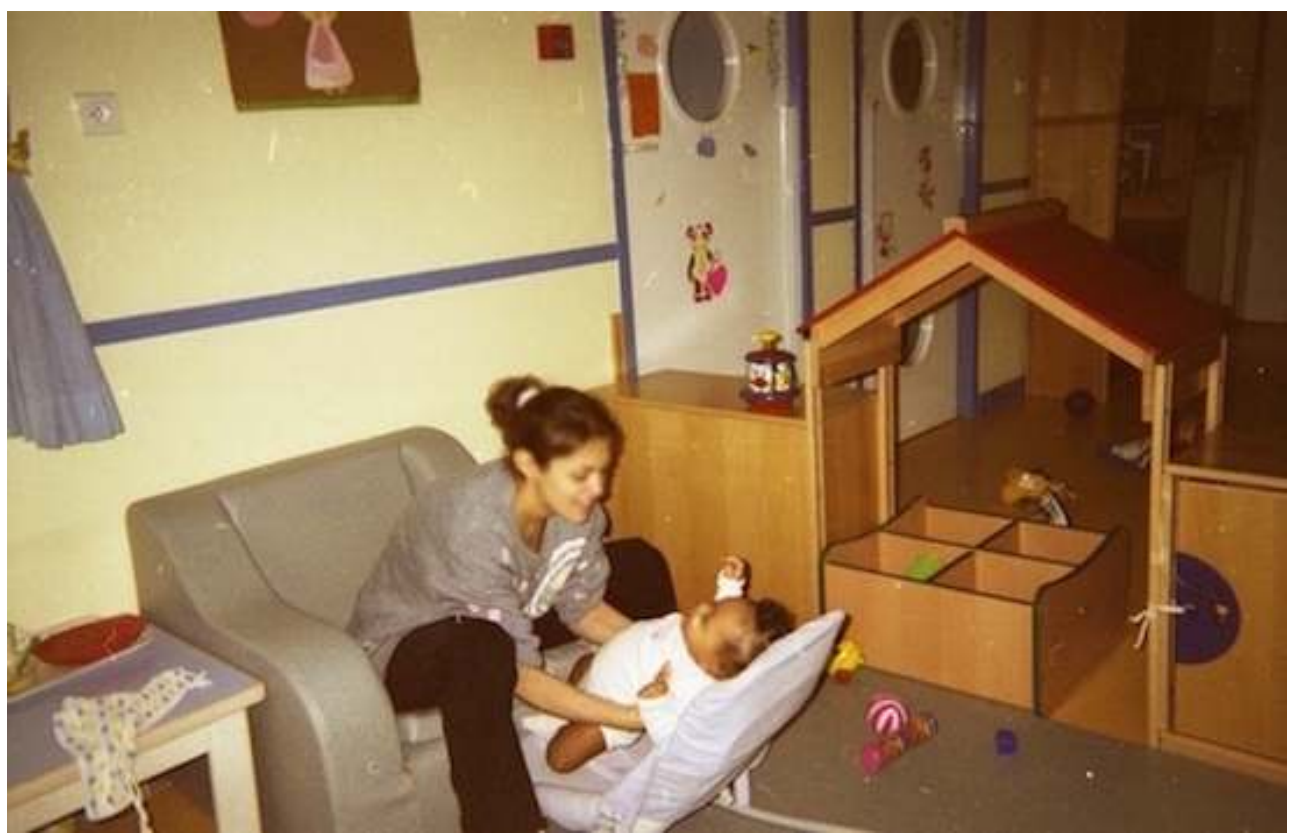

Une autre auxiliaire prend alors également la parole pour expliquer que dans sa crèche les espaces sont séparés par de petites barrières, mais que le loquet est si difficile à ouvrir qu'elles passent leur journée à l'enjamber, parfois avec des enfants dans les bras (Photo 12).

Photographie 12.

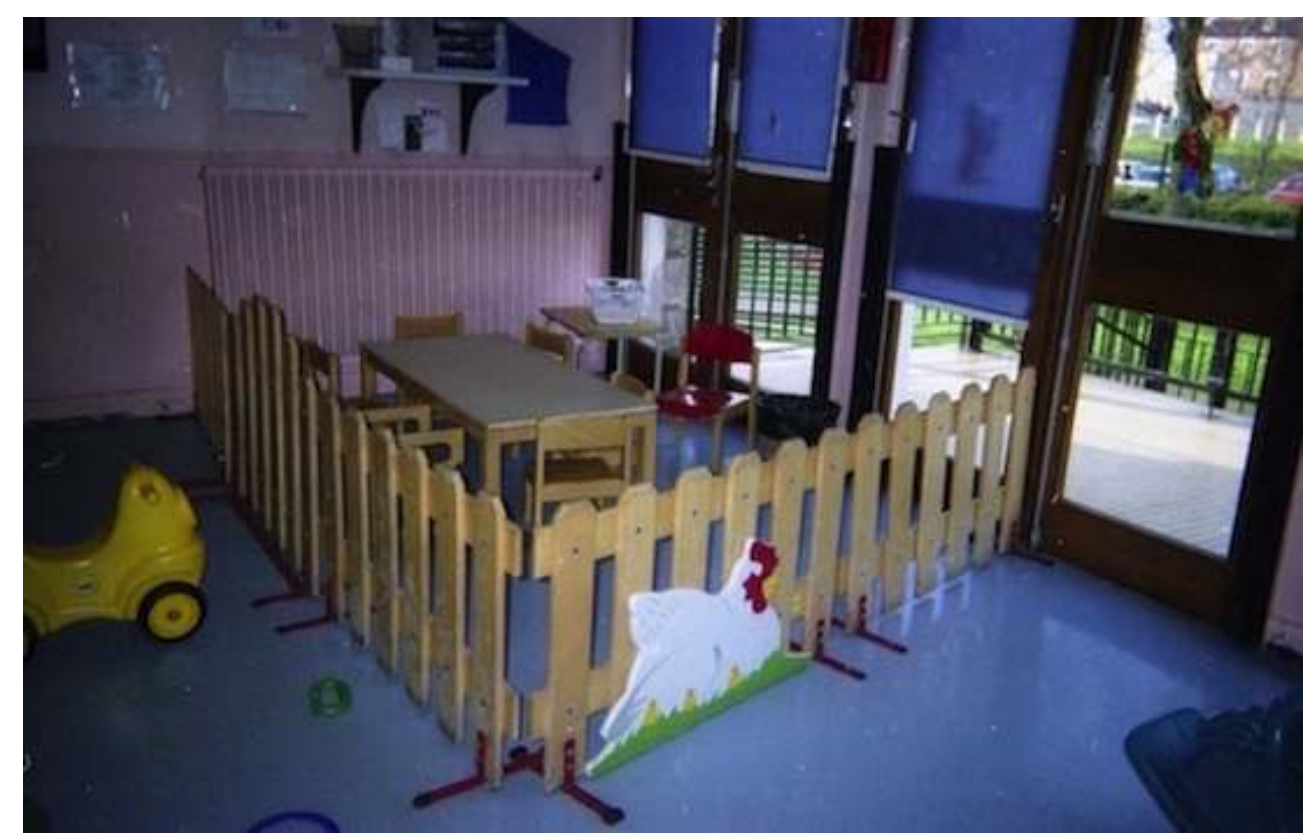




\section{Les compétences au travail}

Le premier souci des auxiliaires est le bien-être de l'enfant et des enfants ensemble. Pour ce faire, elles mettent en place mille et une stratégies pour respecter à la fois leur individualité et le groupe. Elles cherchent ainsi à accompagner l'autonomie des enfants tout en respectant leur rythme, à les responsabiliser, à les laisser prendre des initiatives et à être à leur écoute. En dehors des activités (dessin, peinture, chansons, etc.), elles s'efforcent de laisser place à leur imagination à travers des jeux qu'ils improvisent tout en étant vigilantes.

«Ce petit garçon, il est souvent avec les mallettes de docteur... Cette petite fille, c'est souvent elle qui se fait ausculter, en fait elle est bien dans son corps. Moi j'étais en activité libre avec d'autres enfants, mais en même temps j'observais... Ils se sont donc mis à jouer au docteur et du docteur au bébé et le bébé c'était elle. Le "bébé" a enlevé son pantalon, puis sa culotte, j'ai laissé, je n'ai rien dit, mais à un moment donné j'ai été très surprise, très choquée... Le problème c'est qu'après avoir enlevé son pantalon et sa culotte, d'abord il lui a mis une couche pour faire comme pour les bébés, et plusieurs enfants sont arrivés devant elle... J'ai encore laissé, mais là on n'était plus dans le jeu de la couche, elle avait les jambes écartées, tous regardaient et il y avait un petit garçon qui était très agité, c'est un petit garçon qui n'a pas une vie très équilibrée qu'il faut canaliser beaucoup, et il s'est mis à gesticuler, et là, je me suis retournée et j'ai dit < non >, c'est pas possible, j'ai eu peur de ce qui pouvait se passer. J'ai laissé faire, mais dès que j'ai vu que tout le monde s'était regroupé, je me suis dit < mince et sa pudeur ! >. Et qu'est-ce qui va arriver avec ce petit garçon qui fait ces grands gestes... Donc, je me suis levée, j'ai été voir la petite fille, je l'ai prise à part, je lui ai expliqué : < C'est ton corps, ça n'appartient qu'à toi, je ne veux pas qu'on joue avec ton corps >. Elle m'a regardée, je crois qu'elle a compris... »

Photographie 13.

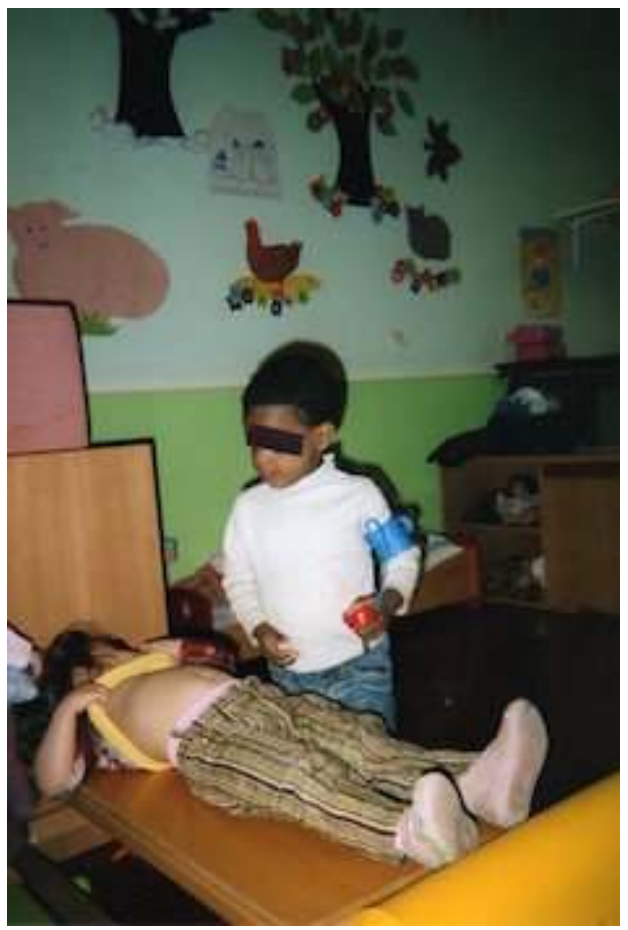

Lorsque la mère est arrivée le soir, Dalila décide de lui raconter l'ensemble de la scène et de son déroulement, ainsi que son ressenti, car pour elle, se promener toute nue 
dans la salle de bain ou être couchée les jambes écartées sous le regard d'un groupe d'enfants ne revêtait pas la même signification.

«Je lui ai raconté du début jusqu'à la fin. Je lui ai dit qu'au début c’était un jeu de bébé, comment ça avait évolué. Et la maman m'a dit : < effectivement ma fille est souvent toute nue dans la maison >. Je lui ai dit: <il n'y a pas de problème, simplement moi ça m'a interpellée parce que là c'est à la crèche et bientôt c'est l'école >. Et ça il fallait que je le dise à la maman. »

Ce dernier exemple, met en avant à la fois le souci de bien-être qu'ont les auxiliaires vis-à-vis des enfants, mais aussi comment la crèche si elle fonctionne comme une véritable équipe peut apporter des solutions. Grâce à la confiance qu'accorde la directrice aux auxiliaires pour leurs initiatives et à l'habitude de laisser circuler les enfants entre les services, Valérie, confrontée à un "grand» qui refuse de dormir depuis son arrivée à la crèche, va essayer de trouver une solution inédite en lien avec sa collègue des bébés.

«Il va toujours chez les bébés parce qu'il n'y a que là qu'il est bien sinon il pleure. Chez les bébés il y a toujours du mouvement, on le sentait un peu angoissé, sa maman nous disait qu'à la maison il ne faisait pas de sieste mais jouait avec des cousins cousines. Je me suis dit je vais rester avec lui et je vais voir, je l'ai laissé chez les bébés et j'ai fait en sorte de le laisser < je vais boire un café et je reviens >. Il est resté à jouer sans faire de bruit, les deux trois plus grands des bébés étaient sur lui, lui apportaient des jouets. Maintenant, c'est lui qui nous prend la main pour aller chez les bébés. On en a discuté avec la psychologue qui a vu la maman, toute la nuit il regarde la télévision de son papa, et la psychologue nous a dit : < réessayez quand même, même s'il va chez les bébés, proposez-lui régulièrement de s'allonger sur son lit >, la directrice nous a dit la même chose en réunion. »

\section{Photographie 14.}

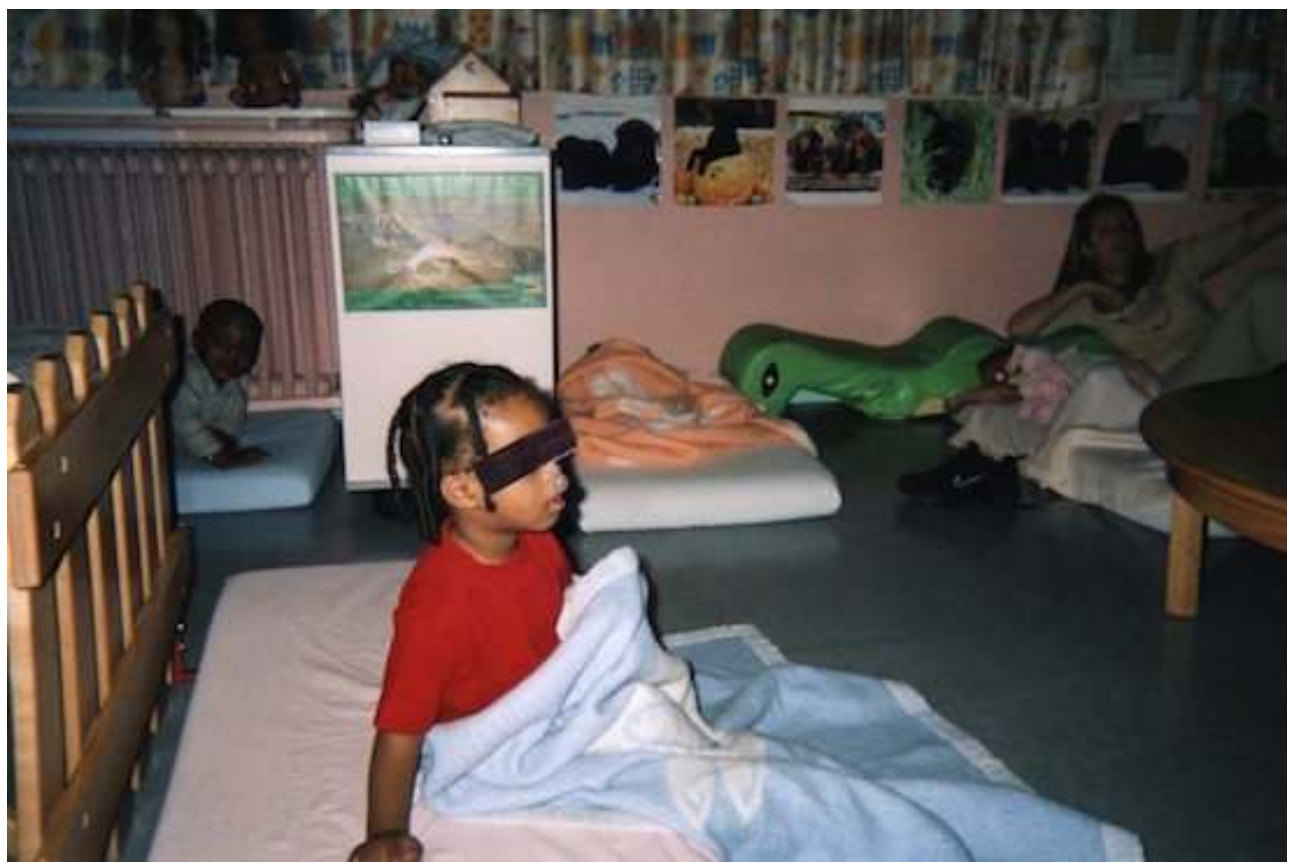

« On lui a proposé, jusqu'au jour où il a accepté de se mettre sur le lit sans pleurer, c'est venu comme ça tout doucement et il s'est allongé. Il nous a rien demandé et maintenant ça fait bien un mois qu'il se met sur le lit, qu'il ne dort pas, qu'il nous écoute parler, discuter, comme ça pendant les siestes. Et depuis quelques temps, ça $\mathrm{y}$ est, il s'allonge et la semaine dernière pour la première fois il s'est endormi, on 
n'en revenait pas. Ca a été très long, mais ce qu'on a remarqué, c'est que depuis qu'on a dit à la maman qu'il acceptait les siestes, il venait plus souvent à la crèche. On avait pris du temps avec la maman pour lui parler de l'école à la rentrée et des horaires de l'école, pour lui faire comprendre qu'il fallait doucement commencer à lui donner un rythme, il a encore le temps mais... »

\section{Conclusion} auxiliaires l'espace et le temps de s'exprimer et d'échanger entre elles, afin qu'elles puissent entreprendre un travail réflexif sur leurs propres appréciations, leurs pratiques et leurs «images ». Il nous semble, au vu des échanges lors des réunions de restitution, que cette démarche collective à partir de leurs photos les a conduites à une meilleure perception de la spécificité et de la valeur de leurs compétences. À partir de leurs images, ce sont les auxiliaires elles-mêmes qui ont pu voir et percevoir leur travail non plus comme un "on fait ça sans s'en rendre compte », mais comme des compétences qui pouvaient être nommées, définies et donc professionnelles. Les discussions autour des images ont fait apparaitre non seulement la connaissance qu'elles ont de chaque enfant pris individuellement dans le groupe, mais les compétences d'intervention dont elles font preuve avec eux à des moments donnés (quand intervenir, comment intervenir) ainsi que les compétences d'adaptation qu'elles ont développé avec les parents.

Cette recherche-action-images a également permis l'inscription de leur travail dans une qualification professionnelle qui s'éloignait d'un «s'occuper des enfants» qui serait à la portée de toute femme, rendant ainsi plus nette à leurs yeux leur identité professionnelle. «Moi », a conclu l'une d'entre elles, « je ne voudrais pas qu'on assimile notre métier à la facilité d'une femme dans ses capacités de maternages. »

\section{BIBLIOGRAPHIE}

Jonas I. (1998), « Vies entre vues. Expériences photographiques », Culture et Proximité, Hors-Série, Février.

Jonas I. et Liane Mozère L. (2006), « Les compétences des auxiliaires de puériculture en images », Rapport pour le service des crèches du CG 93.

Maresca S. (1991), L'autoportrait. Six agricultrices en quête d'image, Toulouse, Presses Universitaires du Mirail / Inra Editions.

Maresca S. et Michaël Meyer M. (2013), Précis de photographie à l'usage des sociologues, Rennes, PUR.

Mozère L. et Jonas I. (dir.), 2011, On « garde » des vaches mais pas les enfants. Paroles d'auxiliaires de puériculture en crèche, Erès. 


\section{NOTES}

1. La sociologue spécialiste de la petite enfance Liane Mozère a disparu le 17 octobre 2013. Sans elle et son souci permanent de laisser la parole aux acteurs, ce projet d'introduire la photographie participative n'aurait probablement jamais vu le jour. Cet article que je signe seule est aussi le sien.

2. Les auxiliaires qui ont suivi la recherche-action venaient d'une trentaine de crèches du département.

3. En 1987, dans le cadre de l'Observatoire Banlieues no 3 du CCI et en collaboration avec l'Agence Faut Voir se déroulait au Centre Georges Pompidou l'exposition Un autre regard sur les jeunes: le leur.

4. De même que les auxiliaires participaient aux réunions sur le mode du volontariat, les directrices de crèches pouvaient permettre ou non que des photos soient réalisées dans leurs crèches. Nous n'avons eu qu'un seul refus sans explication.

5. La recherche-action a démarré en 2004, au début du numérique, il n'était pas possible financièrement d'équiper chaque auxiliaire d'un appareil. De plus, le jetable avec sa pellicule de 24 vues permettait que chaque auxiliaire fasse le même nombre d'images.

\section{RÉSUMÉS}

L'introduction de la photographie dans le cadre d'une recherche-action accompagne et enrichit le travail réflexif des groupes de parole. Parce qu'elle permet une articulation entre le « dire » et le « montrer », elle favorise les échanges entre les acteurs à partir de scènes quotidiennes. Cette possible visibilisation de leurs compétences a généré une véritable réflexion sur la professionnalité de leur action qu'elles ont tendance à présenter comme naturelle.

The use of photographs in a group meeting research contributed to the development of reflexivity and collective learning. Il encouraged exchanges between child care assistants team and researchers. Theses exchanges are based on the difference of how they tell about their job and how they show about it with still pictures. It brinks interesting debate on their professional skils.

\section{INDEX}

Mots-clés : photographie, recherche-action-images, compétences professionnelles

Keywords : photography, pictures, professionnal skils, child care assistant

\section{AUTEUR}

\section{IRÈNE JONAS}

Irène Jonas est sociologue et photographe indépendante. Elle a publié plusieurs articles et un ouvrage sur la photo de famille De l'argentique au numérique. Mort de la photo de famille ? Elle 
poursuit actuellement un travail photographique sur les métiers autour de la pêche dans le Finistère sud. 